\title{
Microfluidics for Advanced Drug Delivery Systems
}

\author{
Reza Riahi ${ }^{1,2, \dagger}$, Ali Tamayol ${ }^{1,2, \dagger}$, Seyed Ali Mousavi Shaegh ${ }^{1,2}$, Amir Ghaemmaghami $^{3}$, \\ Mehmet R. Dokmeci ${ }^{1,2}$, Ali Khademshosseini ${ }^{1,2,4,5,{ }^{*}}$ \\ 'Biomaterials Innovation Research Center, Department of Medicine, Brigham and Women's \\ Hospital, Harvard Medical School, Boston, MA, USA \\ ${ }^{2}$ Harvard-MIT Division of Health Sciences and Technology, Massachusetts Institute of Technology, \\ Cambridge, MA, USA \\ ${ }^{3}$ Division of Immunology, School of Life Sciences, Faculty of Medicine and Health Sciences, \\ University of Nottingham, United Kingdom \\ ${ }^{4}$ Wyss Institute for Biologically Inspired Engineering, Harvard University, Boston, MA, USA \\ ${ }^{5}$ Department of Physics, King Abdulaziz University, Jeddah 21569, Saudi Arabia
}

\begin{abstract}
Considerable efforts have been devoted towards developing effective drug delivery methods. Microfluidic systems, with their capability for precise handling and transport of small liquid quantities, have emerged as a promising platform for designing advanced drug delivery systems. Thus, microfluidic systems have been increasingly used for fabrication of drug carriers or direct drug delivery to a targeted tissue. In this review, the recent advances in these areas are critically reviewed and the shortcomings and opportunities are discussed. In addition, we highlight the efforts towards developing smart drug delivery platforms with integrated sensing and drug delivery components.
\end{abstract}

\section{Introduction}

\begin{abstract}
In recent years, researchers have focused on developing novel drugs as well as strategies for their effective delivery to the target sites to improve the outcome of the treatment process. These strategies aim to enhance the drug bioavailability and specificity, reduce their cytotoxicity, and improve patients' comfort. A considerable portion of the pertinent literature has been devoted to the development of drug or gene carriers [1-3]. These activities range from developing biomaterials that enable controlled release of drugs to discovering antibodies or proteins that ascertain specificity of the site of action. For example, $\mathrm{pH}$ - or temperature-responsive carriers have been synthesized via bulk methods for the release of
\end{abstract}

\footnotetext{
This manuscript version is made available under the CC BY-NC-ND 4.0 license (http://creativecommons.org/licenses/by-nc-nd/4.0/).

$\left.{ }^{[*}\right]$ A. Khademhosseini, Corresponding-Author, alik@ rics.bwh.harvard.edu.

$[\dagger]$ R. Riahi and A. Tamayol contributed equally to this work.

Publisher's Disclaimer: This is a PDF file of an unedited manuscript that has been accepted for publication. As a service to our customers we are providing this early version of the manuscript. The manuscript will undergo copyediting, typesetting, and review of the resulting proof before it is published in its final citable form. Please note that during the production process errors may be discovered which could affect the content, and all legal disclaimers that apply to the journal pertain.
} 
the loaded drug in tissues with lower $\mathrm{pH}$ or higher temperature [4,5]. However, these conventional methods for synthesizing drug carriers may sometimes require large amounts of expensive drugs for fabrication and encapsulation to ensure the desired therapeutic response.

Generating a reproducible release profile requires the fabrication of monodisperse drug carriers, which may not be feasible with conventional methods such as emulsification [6]. Another challenging task using these bulk methods is to fabricate carriers for delivery of several drugs or growth factors with different release profiles, where a precise control over the composition of the employed carriers is required. Localized drug delivery is another active research area in which regular approaches such as hypodermic injection of drug or oral drug delivery are either not capable of controlling the drug release locally or maintaining the drug level over a long period of time [7]. Thus, devising strategies capable of addressing these challenges are important and will have significant clinical implications.

Recent advancements in microtechnologies and microfluidics have impacted various fields including drug discovery, biology, diagnostics, and tissue engineering [8-12]. Microfluidic systems allow precise handling and manipulation of nano- and pico-liter volumes of liquid in a reproducible and tunable fashion. Thus, such systems have been employed for fabrication of complex drug carriers with precise size and composition leading to a predictable and tunable release profile $[13,14]$.

Microfluidic systems can be utilized for active and localized delivery of drugs in preprogrammed and minute quantities. This characteristic facilitates the administration of drugs with short half life or those that carry the risk of cytotoxicity upon systemic administration. Furthermore, some traditional delivery methods such as painful and hazardous injections can benefit from these microtechnologies by fabricating microneedles or needle-free injection systems [15]. Microfluidic systems have been recently designed for transdermal administration of drugs to improve patients' comfort and quality of life [16]. As a result of the recent advancement of biosensing platforms and integration of microfluidics, a new class of drug delivery systems has emerged as promising tools, which can administer drugs on demand to form "smart" systems. These platforms can accurately monitor and analyze therapeutic effects through autonomous feedback loop systems [17].

Integration of engineered tissues and organoids with microfluidics to create organ- and body-ona-chip platforms has created a unique opportunity for preclinical assessment of the efficacy and cytotoxity of drug delivery techniques in vitro. These systems can mimic in vivo microenvironment and allows variation of different parameters in a high throughput manner. Recent advances in development and utilization of such platforms have been discussed elsewhere $[8,18]$ and are not reviewed here. In this short review, we will discuss various microfluidic systems that have been utilized for fabrication of drug carriers. We will also highlight the recent advancements and challenges in microfluidic-based direct drug delivery. In addition, we will discuss the integrated and automated platforms capable of smart drug administration. 


\section{Microfluidics in fabrication of drug carriers}

Developing effective drug carriers is an important aspect of drug delivery research. Such carriers should adjust the release rate, improve the bioavailability, and reduce the side effects of drugs. They also need to improve the uptake of poorly soluble and relatively unstable drugs. In particular, oral delivery carriers should withstand the low $\mathrm{pH}$ of stomach and be small enough or covered with proper markers to pass through the intestinal mucosal barrier and enter blood stream. Reliability and controllability of drug release profile are key factors in the successful application of drug carriers, which depends on their size, shape, uniformity, and composition [19]. For example, nanoparticles with diameter less than $10 \mathrm{~nm}$ are rapidly filtered out in kidneys [20]. On the other hand, larger particles can be recognized by immune system and can be removed through phagocytosis [21].

Microfluidic techniques have been employed for preparation of optimally designed drug carriers with the aim of effective therapeutic response. These techniques enable production of mono dispersed and multifunctional drug carriers with highly tunable physical and chemical properties to promote efficacy of drug transport, release, distribution, and elimination during the course of treatment $[22,23]$. In this section, we will highlight recent advances in fabrication of self-assembled, droplets/emulsions, and non-spherical carriers using microfluidic systems.

\subsection{Self-assembled drug carriers}

Nano to microsize vehicles and drug carriers have been commonly fabricated by selfassembly in microfluidic systems. In this technique, two or multiple streams of various reagents are interfaced and the carriers are formed at the interfacial layer. In general, such self-assembly reactions are achieved through hydrodynamic flow focusing (HFF) as well as passive and active mixing. In hydrodynamic flow focusing a core of carrier solution containing the surfactant mixer is focused by surrounding streams of a miscible buffer in the microchannel (Fig. 1a). The size of synthesized carriers are determined by controlling the mixing rates between different fluid streams, which is governed by geometry of the microchannel, flow rates, and the diffusion coefficient of different miscible streams [20]. Microfluidic mixing has been effectively used for precise self-assembly of polymeric and lipid nanoparticles [20,24], followed by encapsulation or chemical conjugation of active molecules to the synthesized carriers [25]. The size of self-assembled particles created by this method is commonly less than $1 \mu \mathrm{m}$ which facilitate the carrier transport across physiological barriers and minimize the chance of phagocytosis [21]. Abhay et al. demonstrated the self-assembly of highly monodispersed liposomes via a microfluidic HFF method and their size effect on cellular uptake mechanisms [26]. When tested against endocytosis inhibitors, large size liposomes $(97.8-162.1 \mathrm{~nm})$ were subjected to clathrindependent uptake mechanisms, while the smallest liposomes ( $40.6 \mathrm{~nm}$ in diameter) primarily followed a dynamin-dependent pathway. In another example, homogeneous PLGA-PEG copolymer nanoparticles with encapsulated Docetaxel were fabricated using microfluidic nanoprecipitation [25]. The size of the fabricated particles using this method was in the range of $20 \mathrm{~nm}-25 \mathrm{~nm}$, which was smaller than the particles obtained by bulk emulsion precipitation (30-100 nm) [25]. It was also observed that the half life of the particles 
fabricated with microfluidic system was approximately twice of the values for those obtained using the bulk method.

In a hydrodynamic flow focusing microfluidic system, the narrow width of the core stream provides a fast mixing due to small diffusion length scale [25]. The particle size distribution and the rate of particle formation by self-assembly are controlled by diffusive mixing time $\left(\tau_{\operatorname{mix}}=\frac{w^{2}}{9 D} \frac{1}{(1+1 / R)^{2}}\right)$ where $w$ is the channel width, $D$ is the diffusivity of the solvent in the core stream, and $R$ is the ratio of the core stream flow rate to the total flow rate of the surrounding streams [20]. In a fixed geometry, it has been demonstrated that increasing the flow ratio $(R)$ can improve the production rate and increase the average diameter of the produced nanoparticles [27]. To decrease the mixing time and improve the self-assembly process, micromixers have been also used for fabrication of carriers and nanoparticles [28]. In general, such microfluidic mixers are divided into two categories of passive and active systems. In passive mixers, the interfaced streams are mixed by introducing surface microarcitectures or abrupt changes in flow configuration without applying any external forces used in active mixing (Fig. 1b). Lipid nanoparticles (LNPs) encapsulating siRNA, for instance, have been generated using a passive microfluidic mixer resulting in rapid preparation of gene-containing carriers with increased gene silencing efficiency [29].

Despite their advantages, one step HFF and micromixers have not been shown to generate multilayer carriers, which are important for sequential delivery of multiple factors. To address this challenge, diffusion-based microfluidics with sequential reaction steps have been employed for creating multilayer carriers (Fig.1c) [30]. In one study, sequentially fabricated lipoplex nanoplarticles encapsulating Bcl-2 antisense deoxyoligonucleotide (ODN) showed higher level of Bcl-2 antisense uptake in K562 human leukemia cells and more efficient down-regulation of Bcl-2 protein level in comparison with carriers made by bulk mixing methods [30].

Overall, HFF systems and passive micromixers are easy to fabricate and operate for generating particles with relatively uniform size distribution. However, formed particles are usually small $(>1 \mu \mathrm{m})$ and cannot be employed for applications were long term release of drug is desired. Another limitation of fabrication of self-assembled carriers is diffusionlimited mass transfer between the co-laminar streams, which limits the production rate and scalability of the process. Thus, novel designs which benefit from active mixing systems with improved mass transfer rate would enhance the rate of carrier production.

\subsection{Droplet-based carriers}

Droplet-based microfluidics is the most popular carrier synthesizing method that can generate highly reproducible and homogeneous drug-loaded particles, microcapsules, microbubbles, and microgels (Fig. 1d-f) [31,32]. The shear stress combined the interfacial tension between immiscible fluids enable the droplet production, where the particles size can be controlled by adjusting the flow rates, solution viscosity, and surface tension. In comparison with self-assembly methods that were used to generate nanoparticles, particles that are produced by droplet-based microfluidic systems are typically larger (i.e. microscale). Such microcarriers are able to provide high drug loading or drug encapsulation and 
maintain sustained drug release for relatively long periods. Tuning of drug release can also be achieved by tailoring the internal structure of such droplets (e.g double or multiple emulsions) which enables simultaneous delivery of multiple drugs.

Production of single and multiple emulsion-based carriers produced through microfluidic approaches can be achieved using combination of cross-flow, flow focusing, and co-flow configurations. In cross-flowing geometry droplets are generated in dripping regime inside microfluidics system with X-, Y-, or T-junctions. However, flow-focusing [33] and coflowing [34] produce droplets using jetting and dripping modes. Droplet-based microfluidics is versatile and allows the fabrication of monodisperse particles from various polymers such as poly(lactide-co-glycolide) (PLGA), poly(lactic acid) (PLA), alginate, and poly(ethylene glycol) (PEG) [19]. The surface chemistry of the fabricated carriers significantly affects the drug release profile as well as their recognition by the immune system [19]. For example, coating the carriers with a PEG layer can prolong their systemic circulation as well as delay uptake by cells and clearance. Thus, multiple emulsions have been fabricated using multiple flow configurations with opposite wettabilities [35]. In a study, lipid microparticles, were synthesized via droplet-based microfludics through a co-flow dripping configuration and congealing process to solidify the melted lipid [36]. Results demonstrated a narrow size distribution with optimal morphological characteristics (e.g. sphericity, surface smoothness) of the fabricated lipid microparticles. Similarly, microcapsules (aqueous core) and microbubbles (gaseous core) can be generated using the droplet-based microfluidic approach through solidification methods such as evaporation, extraction, and multilayer deposition [37,38]. Microgel-based carriers have also been fabricated by flow-focusing microfluidic systems [39]. These microgels are usually formed from hydrophilic stimuli-responsive polymers and hydrogels with high water content making them promising candidates for drug carriers [40]. In particular, the rapid and reversible changes in their pore size in response to physiochemical stimuli make microgels attractive for smart drug delivery. For instance, poly(N-isopropylacrylamide) (PNIPAM), with polymer chains containing both hydrophilic amide groups and hydrophobic isopropyl groups, is commonly used as thermosensitive microgels triggered by changes in temperature and $\mathrm{pH}$ [41]. Recently, a study was conducted to demonstrate the fabrication of water-actuated microgels-based on microfluidic double emulsion [42]. The generated microgles were able to release encapsulated actives by hydration.

In order to control the size, shape and composition of complex particles containing gene or drug carriers, automated and computer controlled microfluidic platforms with integrated micropumps and/or microvalves have been used (Fig. 1g). Sung et al. fabricated a programmable valve-actuated microfluidic system to generate anisotropic elongated particles with exact length, variable bonding angle, pre-designable size sequence and chemical order [43]. Similar pneumatic microfluidic processors have been implemented for generating large drug carrier for both drug delivery and supramolecular droplet libraries for gene transport [44]. Thus, automated platforms can be used for rapid single- or multi-step carrier synthesis, to minimize unpredictability and enhance flexibility in production of carriers and drug loading. 
Droplet-based microfluidics is the most robust fabrication method for multifunctional drug carriers with tunable size and release profile. However, the main shortcoming of this technique is inability for fabricating nanosize drug carriers as well as the complexity of handling and optimization of fluidic circuit. Regular emulsion-based microfluidic systems offer a low throughput, which can be significantly improved by creating systems containing an array of $\mathrm{T}$ junctions. However, their utilization for industrial scale is still not feasible.

\subsection{Non-spherical carriers and particles}

The microfluidic particle generation techniques that were described in the previous subsections usually result in the generation of spherical particles. However, recent studies have shown that particle shape can affect particles in vivo biodistribution, their uptake mechanisms, and their blood circulation time in human body [45]. Thus, non-spherical particles have attracted an increasing amount of attention for drug delivery investigations. They are able to mimic the compelling properties of natural entities like red blood cells. Their high surface-to-volume ratio also provides more cell membrane attachment for drug delivery. Geng et al. showed that long circulating filomicells with paclitaxel increased the apoptosis rate of tumor cells and effectively reduced the tumor size in mice [46]. In another study, Kolhar et al. demonstrated the delivery of siRNA with needle-shape polymeric nanoparticles in vascular endothelium. The gene silencing efficiency improved with the aspect ratio of the non-spherical particles [47].

Non-spherical particles have been fabricated through the self-assembly and coalescence of spherical building blocks created through emulsion-based systems (Fig. 2a) [48], stretching and deforming droplets in microchannels prior or during their solidification [49], or by flow lithography [50]. The self-assembly and coalescence of droplets have been used to produce various structures such as rod-like, cylindrical, and disc-like particles [48,51]. However the arbitrary control of particle geometry is challenging. Stretch or deformation of produced emulsion droplets is another strategy to form anisotropic particles through solidification of polymer solutions in microfluidic platforms. It has been shown that the shape of particles fabricated by microfluidic emulsion and solvent removal solidification, can be changed from spheres to toroids by controlling the flow rate and the solvent diffusion rate (Fig. 2b) [49]. In this section we focus more on strategies used to fabricate non-spherical particle using flow lithography in microfluidics.

In flow lithography, a solution of photocrosslinkable polymer is flowed through a microchannel and is exposed to a light [50]. A photomask can be used to enable creating particles with a predefined geometry. Depending on the temporal flow rate, flow lithography techniques can be divided in two groups of stop flow and continuous flow lithography. In stop flow system, once the prepolymer solution fills the channel, flow is stopped, and light is illuminated on the channel to create the particles (Fig. 2c) [52]. In continuous systems on the other hand, the light illumination system is continuously turned on and off to create particles [53].

Continuous flow systems have a higher throughput than stop flow systems and production rates of 100 particles per second are achievable. However, both conventional stop flow and continuous flow lithography techniques can only fabricated planar particles. To enable the 
fabrication of 3D particles, a microfluidic system has been interfaced with a two photon polymerizer in which its focal plane could be changed across the depth of the channel (Fig. 2d) [54]. However, this technique has a relatively low throughput, which limits its application for drug delivery.

Flow lithography techniques can be utilized to fabricate both spherical and non-spherical particles and carriers. In comparison to droplet-based systems, flow lithography platforms are easier to operate due to presence of one phase in the fluidic channel. A key challenge associated with flow lithography methods is their low throughput. Moreover, light sensitive drugs cannot be incorporated into the carriers fabricated by flow lithography. The smallest size of particles fabricated using lithography techniques is limited to the resolution of the illumination system; thus, fabrication of sub-micron size particles is challenging.

\section{Microfluidic platforms for direct drug delivery}

In addition to the possibility of fabricating complex drug carriers, microfluidic systems can be utilized for direct delivery of active molecules [55]. Such systems are capable of efficiently transporting drugs to a targeted site to increase the local availability of the drug and to reduce the side effects caused by the interaction of the drug with other organs and tissues. In addition, microfluidic systems have been successfully employed for the so called transdermal delivery, which is direct drug delivery across the skin. The goal of these systems, that utilize a needle or an array of microneedles, is to transfer the drug across the skin (epidermis) barrier. In this section we will discuss the advantages of microfluidic systems employed for localized and transdermal drug delivery.

\subsection{Localized drug delivery}

There are a number of techniques employed for localized drug delivery such as the use of drug loaded polymers and the use of microfluidic implantable devices [56]. Microfluidic systems are capable of using convective forces for on demand drug release, which differentiate them from other diffusion-based local delivery platforms with continuous and non-uniform release profile. Thus, microfluidic platforms are able to control the release profile. The microfluidic platforms are usually comprised of a pump or actuator, a valve, a drug reservoir, and a membrane for controlling the release rate. The simplest approach is to physically compress the reservoir to force the contained drug out. Lo et al. engineered a microfluidic drug delivery device with a refillable drug reservoir for treating ocular diseases [57]. The device included a PDMS-based check valve to control the release rate of the drug after pressurizing the reservoir manually (Fig. 3a-b). The flow rate of the device was varying from $0.61 \mu \mathrm{l} / \mathrm{s}$ for $250 \mathrm{mmHg}$ of applied pressure to $1.57 \mu \mathrm{l} / \mathrm{s}$ for $500 \mathrm{mmHg}$. This variation in the drug release rate in response to the actuation pressure could be a limitation of this platform, especially if the system is finger actuated [57]. Pressurizing the drug reservoir in implantable devices is a key challenge and researchers have tried to design various easy to implement mechanisms for achieving this aim. Chung et al. incorporated two electrodes on the top and bottom surfaces of the drug reservoir covered with a membrane (Fig. 3c) [58]. To release the contained drug, an electrical potential was applied via two electrodes triggering two chemical reactions which formed gas bubbles to break the membrane and push out the 
drug. A similar system, was implanted into a Manduca sexta moth to actively control the moth behavior (movement) through chemical delivery (Fig. 3d) [59].

Another method demonstrated by Elman et al. was to use a microfluidic platform with a reservoir covered with a silicon nitride membrane (Fig. 3e-f) [60]. The reservoir included a heating module to create a film boiling within the entrapped liquid to break the brittle membrane and release the drug. This system is effective for the rapid delivery and can be used for emergency applications with life threatening conditions. However, this system cannot be used for continuous drug delivery over a long period of time. Moreover, the generated heat limits its use for thermally unstable drugs and growth factors.

Drug delivery platforms with magnetic actuation are promising candidates for localized drug delivery as magnetic field can easily penetrate the body. Pirmoradi et al. developed a microfluidic system comprised of a drug reservoir sealed by a magnetic responsive iron oxide doped PDMS hanging membrane with a laser drilled aperture (Fig. 3g) [61]. After applying a magnetic field, the membrane was deformed pushing out the drug through the membrane. The challenge with such a system is the inconsistency of release rate in each cycle for cyclic drug delivery.

Overall, the localized microfluidic platform for direct drug delivery to the injury site holds a great promise. However, in many applications, such platforms should be degradable in a way that after successful operation they could be resorbed without the need for a secondary surgery. Moreover, their mechanical characteristics should match those of the surrounding tissue to prevent interference with the tissue function and improve patients' comfort. Another important research area, is to develop an easy-to-operate actuation mechanism combined with an effective sub-nanoliter scale flow regulating system that enable long term and on demand administration of drugs. A key challenge to overcome for designing longlasting drug delivery platforms is the immune and inflammatory response and the interaction of the tissues with the device. Inflammation can deteriorate the performance of the device and in severe cases may lead to device rejection and the need for its removal. Fibrosis may also affect the device operation as it can block the nozzles or restrain the movement of mechanical components. Thus, coating the construct with anti-fibrotic factors may prolong the reliability of the system over the course of treatment.

\subsection{Transdermal drug delivery}

Drugs can be administered to the patient's body through a number of routes. Skin is an easy to access organ for delivering active molecules; however, it forms a strong barrier that protects the body from outside environment. Thus, developing transdermal drug delivery platforms has attracted a lot of attention. Recently, arrays of miniaturized microneedles have been developed to penetrate the epidermis without disrupting the nerve-rich regions; thus, enabling painless drug delivery in comparison to conventional hypodermal delivery systems. These microneedles have different forms such as: i) solid microneedles disrupting the epidermis barrier, ii) microneedles coated with drug, iii) dissolvable microneedles releasing drug in a gradual pace, and iv) hollow microneedles enabling convective drug transport across the barrier (Fig. 3h). In this short review, we primarily discuss the fourth category. 
In a study, McAllister et al. microfabricated tapered microneedles from a range of materials including metals, silicon, and glass (Fig. 3j) [62]. They used the microneedles for insulin injection and demonstrated the effectiveness of the strategy in lowering blood sugar level. A comprehensive review of the pertinent literature can be found elsewhere [63]. These microneedles can be combined with actuation systems such as piezoelectric systems, spring, pressurized gas, and microgear pump for active and preprogrammed drug delivery [64].

These microneedles have also been integrated with sensing systems to improve their sensitivity through breaching the epidermal barrier. In a notable study, Yu et al. fabricated silicon microneedles and used them for electrocardiography (ECG) [65]. The microneedles penetrated through the skin and reduced the electrode-skin-electrode impedance. Moreover, they could inject a $\mathrm{NaCl}$ solution through the needles as an electrolyte. Their results indicated a significant improvement of signal-to-noise ratio of ECG measurement by electrodes with microneedles as compared to electrodes with flat surface.

Transdermal microfluidic drug delivery platforms one day replace the hypodermic needles and can also be integrated with sensing platforms for designing multifunctional systems. Also, such platforms can be combined with bioinspired reversible dry adhesives for creating needles with high adhesion force while being easy to remove. However, susceptibility to clogging and fibrosis can affect their normal function and strategies should be devised to prevent it. Another challenge that may affect the long term use of transdermal drug delivery devices is bacterial infection as the skin barrier is breached for the duration of their use. Thus, special attention should be paid to designing systems with bacterial inhibition characteristics.

\section{Smart and autonomous integrated microfluidic systems for drug delivery}

Smart drug delivery devices are mostly used to keep drugs at a desirable level in body to avoid the need for frequent dose [67]. Although there are a great number of investigations focusing on integrated (implantable) microfluidic devices for drug delivery, there is a huge need to convert such devices to self-regulating smart and autonomous drug delivery systems [64]. Such devices are highly desirable for treating chronic diseases such as diabetes and rheumatoid arthritis [68], where continuous and controlled drug delivery is the key to successful treatment of patients.

A smart and autonomous microfluidic drug delivery device is mainly made of four miniaturized building blocks including i) a drug reservoir; ii) a controllable actuator to inject therapeutic levels of the drug to body, iii) a (bio)sensing module and its signal processor for measuring a marker or an analyte and controlling drug release profile in a closed-loop arrangement, and iv) a power source for sensing and actuation processes. Various designs of actuators for drug injection integrated in drug reservoirs were discussed in the previous section. (Bio)sensors can be potentially designed to be integrated with bio-responsive materials for measurement of a specific drug concentration or a particular marker molecule of the disease being cured [68,69]. 
Tsai et al. fabricated a glucose sensor by immobilizing glucose oxidase on a hydrogel (Fig. 4a) [68]. In order to preserve the sensitivity of the sensor, it was mounted on the lid of a microfabricated vial to protect it from surrounding environmental conditions (e.g. the human Body). Upon applying $800 \mathrm{mV}$ potential to the lid made of polymer/gold, it could be opened to expose the sensor to the stream of a marker or analyte. In addition, the lid can be mounted on a microfabricated drug reservoir to act as a microvalve to open and close the drug reservoir for a closed-loop arrangement with the sensor to regulate therapeutic level of a drug [68].

In a notable study Son et al. developed a wearable patch for diagnosis and therapy of movement disorders by controlled delivery of therapeutic agents (Fig. 4b) [70]. M-silica nanoparticles with drug loaded nanopores were used as vehicles for adsorption and delivery of drugs. Nanoparticles were transfer-printed onto the sticky side of a patch with an electroresistive heater/sensor using a PDMS stamp. Upon heating the patch, the pharmacological agents loaded in the nanoparticles can diffuse transdermally. The temperature sensor was able to monitor the maximum temperature on the skin in order to protect it from getting burnt.

Key challenges for making smart microfluidic drug delivery devices are revolving around fabrication and integration of miniaturized components in a closed-loop arrangement, and developing sensors that can operate reliably over long periods of time. Different methods for drug pumping have been developed for implanted microfluidic drug delivery devices [64]. One key challenge is to actuate the micropump based on a feedback signal coming from the measuring sensors in order to maintain the drug concentration at a therapeutic level. Also, the measuring sensors should have very stable operation in terms of sensitivity and selectivity over the period of implantation time. The sensors can be potentially designed to be integrated with bio-responsive materials for measurement of a specific drug concentration or a particular marker molecule of the disease being cured [68,71]. Further details related to miniaturized implantable drug delivery devices can be found elsewhere $[68,72]$.

\section{Challenges, concluding remarks, and future directions}

During the past decades, the design of microfluidic systems and their functionality have substantially improved and microfluidic platforms have made their way to various areas of medicine including drug delivery. Microfluidic systems can enable fabrication of sophisticated drug carriers with uniform sizes in the range of hundreds of $\mathrm{nm}$ to several $\mu \mathrm{m}$. In addition such systems are able to design and fabricate drug carriers with preprogrammed release profile. We believe that the majority of future advances in this area will be devoted to the utilization of advanced polymers for fabrication of controllable and multifunctional drug carriers.

Microfluidic systems have also been employed for the direct and localized delivery of drug to target sites. These systems are capable of delivery of exact and small quantity of drug doses reducing the need for using high concentrations of drugs with significant side effects. One future direction of such systems could be the fabrication of biodegradable drug delivery platforms, where the implanted system can be resorbed in vivo without the need for a 
secondary surgery for its removal. The associated challenge is to have a controllable life span for these drug delivery systems. The device should operate with optimum performance during the course of treatment without degradation. When the treatment is over, the device should start to degrade in the body to avoid possible surgical processes for device removal. To address this issue, the device components may be made from biodegradable materials that are coated with a protective layer to prevent degradation. Whenever the treatment period ends, the coating layer will be removed or degraded by a stimulus so the drug delivery device can be exposed to enzymes of body fluids for rapid degradation process. Another area that is expected to receive significant attention is the combination of sensing platforms and microfluidic systems for drug delivery. The utilization of polymeric substrates in fabricating electronic systems and sensors has paved the road for fabrication of flexible and bioresorbable electronics [73]. Biodegradable batteries and biofuel cells extracting power from body fluids might be a future source to empower such devices. Their main challenge for integration with drug delivery devices is to have long-term performance stability at human body conditions. These electronic platforms can be integrated with microfluidic systems or responsive drug carriers for automated and on demand drug administration. It is expected that with the emergence of smart systems it will be possible to employ them for sensing different biomarkers (e.g. analytes or metabolites) in the body and administer the appropriate therapeutics accordingly. Such systems will revolutionize the patient management strategies for the treatment of various diseases. A major challenge, however, is to develop a monitoring strategy that not only can control the performance of an implanted smart drug delivery device but also trigger a secondary method for situations where the device failure occurs. This is particularly of great importance for those life-preserving devices that sustain patient life.

Integration of microfluidic systems for direct drug delivery or fabrication of drug carriers with recently emerging organ-on-a-chip platforms allows cost effective studies on the efficacy of various drug delivery systems. Moreover, it is expected that these integrated miniaturized systems can fill the gap between the animal studies and human clinical trials.

\section{Acknowledgements}

The authors declare no conflict of interests in this work. A.K. acknowledges funding from the National Science Foundation (EFRI-1240443), the office of Naval Research Young National Investigator Award, the National Institutes of Health (HL092836, DE019024, EB012597, AR057837, DE021468, HL099073, EB008392), and the Presidential Early Career Award for Scientists and Engineers (PECASE).

\section{References}

1. Zhang Y, Chan HF, Leong KW: Advanced materials and processing for drug delivery: The past and the future. Advanced drug delivery reviews 2013, 65:104-120. [PubMed: 23088863]

2. Couvreur P: Nanoparticles in drug delivery: Past, present and future. Advanced drug delivery reviews 2013, 65:21-23. [PubMed: 22580334]

3. Singh A, Agarwal R, Diaz-Ruiz CA, Willett NJ, Wang P, Lee LA, Wang Q, Guldberg RE, García AJ: Nanoengineered Particles for Enhanced Intra-Articular Retention and Delivery of Proteins. Advanced Healthcare Materials 2014:

4. Guo M, Que C, Wang C, Liu X, Yan H, Liu K: Multifunctional superparamagnetic nanocarriers with folate-mediated and $\mathrm{pH}$-responsive targeting properties for anticancer drug delivery. Biomaterials 2011, 32:185-194. [PubMed: 21067808] 
5. Cheng R, Meng F, Deng C, Klok H-A, Zhong Z: Dual and multi-stimuli responsive polymeric nanoparticles for programmed site-specific drug delivery. Biomaterials 2013, 34:3647-3657. [PubMed: 23415642]

6. Duncanson WJ, Lin T, Abate AR, Seiffert S, Shah RK, Weitz DA: Microfluidic synthesis of advanced microparticles for encapsulation and controlled release. Lab on a Chip 2012, 12:21352145. [PubMed: 22510961]

7. Prausnitz MR, Langer R: Transdermal drug delivery. Nature Biotechnology 2008, 26:1261-1268.

8. Bhise NS, Ribas J, Manoharan V, Zhang YS, Polini A, Massa S, Dokmeci MR, Khademhosseini A: Organ-on-a-chip platforms for studying drug delivery systems. J Control Release 2014, 190:82-93. [PubMed: 24818770] * This paper highlights the importance of microfludic integration with 3D tissue model to create organ-on-chip platforms.

9. Tamayol A, Akbari M, Annabi N, Paul A, Khademhosseini A, Juncker D: Fiber-based tissue engineering: Progress, challenges, and opportunities. Biotechnology Advances 2013, 31:669-687. [PubMed: 23195284]

10. Safavieh R, Juncker D: Capillarics: pre-programmed, self-powered microfluidic circuits built from capillary elements. Lab on a Chip 2013, 13:4180-4189. [PubMed: 23978958]

11. Bratt-Leal AM, Nguyen AH, Hammersmith KA, Singh A, McDevitt TC: A microparticle approach to morphogen delivery within pluripotent stem cell aggregates. Biomaterials 2013, 34:7227-7235. [PubMed: 23827184]

12. Avesar J, Arye TB, Levenberg S: Frontier microfluidic techniques for short and longterm single cell analysis. Lab on a Chip 2014, 14:2161-2167. [PubMed: 24671389]

13. Wu J, Kong T, Yeung KWK, Shum HC, Cheung KMC, Wang L, To MKT: Fabrication and characterization of monodisperse PLGA-alginate core-shell microspheres with monodisperse size and homogeneous shells for controlled drug release. Acta Biomaterialia 2013, 9:7410-7419.

[PubMed: 23535235] * PLGA-alginate core-shell microspheres exhibited sigmoid release patterns of drug in vitro.

14. Kong T, Wu J, To M, Wai Kwok Yeung K, Cheung Shum H, Wang L: Droplet based microfluidic fabrication of designer microparticles for encapsulation applications. Biomicrofluidics 2012, 6:-.

15. Kochhar JS, Goh WJ, Chan SY, Kang L: A simple method of microneedle array fabrication for transdermal drug delivery. Drug development and industrial pharmacy 2013, 39:299-309. [PubMed: 22519721]

16. Kochhar JS, Anbalagan P, Shelar SB, Neo JK, Iliescu C, Kang L: Direct microneedle array fabrication off a photomask to deliver collagen through skin. Pharmaceutical Research 2014:1-11.

17. Jivani RR, Lakhtaria GJ, Patadiya DD, Patel LD, Jivani NP, Jhala BP: Biomedical microelectromechanical systems (BioMEMS): Revolution in drug delivery and analytical techniques. Saudi Pharmaceutical Journal 2013.

18. Polini A, Prodanov L, Bhise NS, Manoharan V, Dokmeci MR, Khademhosseini A: Organs-on-achip: a new tool for drug discovery. Expert Opinion on Drug Discovery 2014, 9:335-352. [PubMed: 24620821]

19. Champion JA, Katare YK, Mitragotri S: Particle shape: A new design parameter for micro- and nanoscale drug delivery carriers. Journal of Controlled Release 2007, 121:3-9. [PubMed: 17544538]

20. Lo CT, Jahn A, Locascio LE, Vreeland WN: Controlled Self-Assembly of Monodisperse Niosomes by Microfluidic Hydrodynamic Focusing. Langmuir 2010, 26:8559-8566. [PubMed: 20146467]

21. Owens DE III, Peppas NA: Opsonization, biodistribution, and pharmacokinetics of polymeric nanoparticles. International journal of pharmaceutics 2006, 307:93-102. [PubMed: 16303268]

22. Gañán-Calvo AM, Montanero JM, Martín-Banderas L, Flores-Mosquera M: Building functional materials for health care and pharmacy from microfluidic principles and Flow Focusing. Advanced drug delivery reviews 2013, 65:1447-1469. [PubMed: 23954401]

23. Tsui JH, Lee W, Pun SH, Kim J, Kim D-H: Microfluidics-assisted in vitro drug screening and carrier production. Advanced drug delivery reviews 2013, 65:1575-1588. [PubMed: 23856409]

24. Basta T, Wu H-J, Morphew MK, Lee J, Ghosh N, Lai J, Heumann JM, Wang K, Lee YC, Rees DC, et al.: Self-assembled lipid and membrane protein polyhedral nanoparticles. Proceedings of the 
National Academy of Sciences 2014, 111:670-674.** MPPNs provide a starting point for the structural analysis of membrane proteins in a phospholipid environment.

25. Karnik R, Gu F, Basto P, Cannizzaro C, Dean L, Kyei-Manu W, Langer R, Farokhzad OC: Microfluidic Platform for Controlled Synthesis of Polymeric Nanoparticles. Nano Letters 2008 , 8:2906-2912. [PubMed: 18656990]

26. Andar A, Hood R, Vreeland W, DeVoe D, Swaan P: Microfluidic Preparation of Liposomes to Determine Particle Size Influence on Cellular Uptake Mechanisms. Pharmaceutical Research 2014, 31:401-413. [PubMed: 24092051]

27. Lim J-M, Bertrand N, Valencia PM, Rhee M, Langer R, Jon S, Farokhzad OC, Karnik R: Parallel microfluidic synthesis of size-tunable polymeric nanoparticles using 3D flow focusing towards in vivo study. Nanomedicine: Nanotechnology, Biology and Medicine 2014, 10:401-409.**

Parallelization of nanoparticles synthesis using a multilayer microfluidic system can improve the production rate.

28. Serra CA, Khan IU, Cortese B, de Croon MHJM, Hessel V, Ono T, Anton N, Vandamme T: Microfluidic Production of Micro- and Nanoparticles In Encyclopedia of Polymer Science and Technology. Edited by: John Wiley \& Sons, Inc.; 2002.

29. Belliveau NM, Huft J, Lin PJC, Chen S, Leung AKK, Leaver TJ, Wild AW, Lee JB, Taylor RJ, Tam YK, et al.: Microfluidic Synthesis of Highly Potent Limit-size Lipid Nanoparticles for In Vivo Delivery of siRNA. Mol Ther Nucleic Acids 2012, 1:e37. [PubMed: 23344179]

30. Koh CG, Zhang X, Liu S, Golan S, Yu B, Yang X, Guan J, Jin Y, Talmon Y, Muthusamy N, et al.: Delivery of antisense oligodeoxyribonucleotide lipopolyplex nanoparticles assembled by microfluidic hydrodynamic focusing. Journal of Controlled Release 2010, 141:62-69. [PubMed: 19716852]

31. Zhao C-X: Multiphase flow microfluidics for the production of single or multiple emulsions for drug delivery. Advanced drug delivery reviews 2013, 65:1420-1446. [PubMed: 23770061]

32. Mou C-L, Ju X-J, Zhang L, Xie R, Wang W, Deng N-N, Wei J, Chen Q, Chu L-Y: Monodisperse and Fast-Responsive Poly(N-isopropylacrylamide) Microgels with Open-Celled Porous Structure. Langmuir 2014, 30:1455-1464. [PubMed: 24437526]

33. Anna SL, Bontoux N, Stone HA: Formation of dispersions using "flow focusing" in microchannels. Applied Physics Letters 2003, 82:364-366.

34. Utada AS, Lorenceau E, Link DR, Kaplan PD, Stone HA, Weitz DA: Monodisperse Double Emulsions Generated from a Microcapillary Device. Science 2005, 308:537-541. [PubMed: 15845850]

35. Teh S-Y, Khnouf R, Fan H, Lee AP: Stable, biocompatible lipid vesicle generation by solvent extraction-based droplet microfluidics. Biomicrofluidics 2011, 5:-.

36. Capretto L, Mazzitelli S, Nastruzzi C: Design, production and optimization of solid lipid microparticles (SLM) by a coaxial microfluidic device. Journal of Controlled Release 2012, 160:409-417. [PubMed: 22542700]

37. Chen PW, Erb RM, Studart AR: Designer Polymer-Based Microcapsules Made Using Microfluidics. Langmuir 2011, 28:144-152. [PubMed: 22118302]

38. Priest C, Quinn A, Postma A, Zelikin AN, Ralston J, Caruso F: Microfluidic polymer multilayer adsorption on liquid crystal droplets for microcapsule synthesis. Lab on a Chip 2008, 8:21822187. [PubMed: 19023485]

39. Tumarkin E, Kumacheva E: Microfluidic generation of microgels from synthetic and natural polymers. Chemical Society Reviews 2009, 38:2161-2168. [PubMed: 19623340]

40. Jagadeesan D, Nasimova I, Gourevich I, Starodubtsev S, Kumacheva E: Microgels for the Encapsulation and Stimulus-Responsive Release of Molecules with Distinct Polarities. Macromolecular Bioscience 2011, 11:889-896. [PubMed: 21504069]

41. Shah RK, Kim J-W, Agresti JJ, Weitz DA, Chu L-Y: Fabrication of monodisperse thermosensitive microgels and gel capsules in microfluidic devices. Soft Matter 2008, 4:2303-2309.

42. Huang S, Zeng S, He Z, Lin B: Water-actuated microcapsules fabricated by microfluidics. Lab on a Chip 2011, 11:3407-3410. [PubMed: 21881661] 
43. Sung KE, Vanapalli SA, Mukhija D, McKay HA, Mirecki Millunchick J, Burns MA, Solomon MJ: Programmable Fluidic Production of Microparticles with Configurable Anisotropy. Journal of the American Chemical Society 2008, 130:1335-1340. [PubMed: 18166053]

44. Wang H, Liu K, Chen K-J, Lu Y, Wang S, Lin W-Y, Guo F, Kamei K-i, Chen Y-C, Ohashi M, et al.: A Rapid Pathway Toward a Superb Gene Delivery System: Programming Structural and Functional Diversity into a Supramolecular Nanoparticle Library. ACS Nano 2010, 4:6235-6243. [PubMed: 20925389]

45. Mathaes R, Winter G, Besheer A, Engert J: Non-spherical micro- and nanoparticles: fabrication, characterization and drug delivery applications. Expert Opin Drug Deliv 2014:1-12.

46. Geng Y, Dalhaimer P, Cai S, Tsai R, Tewari M, Minko T, Discher DE: Shape effects of filaments versus spherical particles in flow and drug delivery. Nature Nanotechnology 2007, 2:249-255.

47. Kolhar P, Anselmo AC, Gupta V, Pant K, Prabhakarpandian B, Ruoslahti E, Mitragotri S: Using shape effects to target antibody-coated nanoparticles to lung and brain endothelium. Proceedings of the National Academy of Sciences 2013, 110:10753-10758.

48. Studart AR, Shum HC, Weitz DA: Arrested Coalescence of Particle-coated Droplets into Nonspherical Supracolloidal Structures $\dagger$. The Journal of Physical Chemistry B 2009, 113:39143919. [PubMed: 19673138]

49. Wang B, Shum HC, Weitz DA: Fabrication of Monodisperse Toroidal Particles by Polymer Solidification in Microfluidics. ChemPhysChem 2009, 10:641-645. [PubMed: 19185032]

50. Annabi N, Tamayol A, Uquillas JA, Akbari M, Bertassoni LE, Cha C, Camci-Unal G, Dokmeci MR, Peppas NA, Khademhosseini A: 25th Anniversary Article: Rational Design and Applications of Hydrogels in Regenerative Medicine. Advanced Materials 2014, 26:85-124. [PubMed: 24741694]

51. Shum HC, Abate AR, Lee D, Studart AR, Wang B, Chen C-H, Thiele J, Shah RK, Krummel A, Weitz DA: Droplet Microfluidics for Fabrication of Non-Spherical Particles. Macromolecular Rapid Communications 2010, 31:108-118. [PubMed: 21590882]

52. Panda P, Ali S, Lo E, Chung BG, Hatton TA, Khademhosseini A, Doyle PS: Stop-flow lithography to generate cell-laden microgel particles. Lab on a Chip 2008, 8:1056-1061. [PubMed: 18584079] * Stop-flow litography is used to synthesize large numbers of cell encapsulated hydrogels in a continuous manner for generating 3Dtissue construct for drug delivery.

53. Dendukuri D, Pregibon DC, Collins J, Hatton TA, Doyle PS: Continuous-flow lithography for high-throughput microparticle synthesis. Nature materials 2006, 5:365-369. [PubMed: 16604080]

54. Laza SC, Polo M, Neves AAR, Cingolani R, Camposeo A, Pisignano D: Two-Photon Continuous Flow Lithography. Advanced Materials 2012, 24:1304-1308. [PubMed: 22302705]

55. LaVan DA, McGuire T, Langer R: Small-scale systems for in vivo drug delivery. Nature Biotechnology 2003, 21:1184-1191.

56. Nagai N, Kaji H, Onami H, Katsukura Y, Ishikawa Y, Nezhad ZK, Sampei K, Iwata S, Ito S, Nishizawa M: A platform for controlled dual-drug delivery to the retina: Protective effects against light-induced retinal damage in rats. Advanced Healthcare Materials 2014.

57. Lo R, Li P-Y, Saati S, Agrawal R, Humayun M, Meng E: A passive MEMS drug delivery pump for treatment of ocular diseases. Biomedical Microdevices 2009, 11:959-970. [PubMed: 19396548]

58. Chung A, Huh Y, Erickson D: A robust, electrochemically driven microwell drug delivery system for controlled vasopressin release. Biomedical Microdevices 2009, 11:861-867. [PubMed: 19353273]

59. Chung A, Cordovez B, Jasuja N, Lee D, Huang X, Erickson D: Implantable microfluidic and electronic systems for insect flight manipulation. Microfluidics and Nanofluidics 2012, 13:345352.

60. Elman NM, Ho Duc HL, Cima MJ: An implantable MEMS drug delivery device for rapid delivery in ambulatory emergency care. Biomedical Microdevices 2009, 11:625-631. [PubMed: 19169826]

61. Pirmoradi FN, Jackson JK, Burt HM, Chiao M: A magnetically controlled MEMS device for drug delivery: design, fabrication, and testing. Lab on a Chip 2011, 11:3072-3080. [PubMed: 21860883]

62. McAllister DV, Wang PM, Davis SP, Park J-H, Canatella PJ, Allen MG, Prausnitz MR: Microfabricated needles for transdermal delivery of macromolecules and nanoparticles: fabrication 
methods and transport studies. Proceedings of the National Academy of Sciences 2003, 100:13755-13760.

63. van der Maaden K, Jiskoot W, Bouwstra J: Microneedle technologies for (trans)dermal drug and vaccine delivery. Journal of Controlled Release 2012, 161:645-655. [PubMed: 22342643]

64. Nguyen N-T, Shaegh SAM, Kashaninejad N, Phan D-T: Design, fabrication and characterization of drug delivery systems based on lab-on-a-chip technology. Advanced drug delivery reviews 2013, 65:1403-1419. [PubMed: 23726943] * This paper reviews microfluidic platforms based on drug delivery at cellular, tissue and organ levels.

65. Yu LM, Tay FEH, Guo DG, Xu L, Yap KL: A microfabricated electrode with hollow microneedles for ECG measurement. Sensors and Actuators A: Physical 2009, 151:17-22.

66. Kim Y-C, Park J-H, Prausnitz MR: Microneedles for drug and vaccine delivery. Advanced drug delivery reviews 2012, 64:1547-1568. [PubMed: 22575858]

67. Kasagana VN, karumuri MT: Recent Advances in Smart Drug Delivery Systems. Int J Nov Drug Deliv Tech 2011, 1:201-207.

68. Tsai H-KA, Moschou EA, Daunert S, Madou M, Kulinsky L: Integrating Biosensors and Drug Delivery: A Step Closer Toward Scalable Responsive Drug-Delivery Systems. Advanced Materials 2009, 21:656-660.

69. áAndrew Lyon L: Bioresponsive hydrogels for sensing applications. Soft Matter 2009, 5:29-35.

70. Son D, Lee J, Qiao S, Ghaffari R, Kim J, Lee JE, Song C, Kim SJ, Lee DJ, Jun SW: Multifunctional wearable devices for diagnosis and therapy of movement disorders. Nature Nanotechnology 2014, 9:397-404.** Wearable patch is used to diagnose and treat movement disorders by controlled delivery of therapeutic agents.

71. Hendrickson GR, Andrew Lyon L: Bioresponsive hydrogels for sensing applications. Soft Matter 2009, 5:29-35.

72. Tng DJH, Hu R, Song P, Roy I, Yong K-T: Approaches and Challenges of Engineering Implantable Microelectromechanical Systems (MEMS) Drug Delivery Systems for in Vitro and in Vivo Applications. Micromachines 2012, 3:615-631.

73. Najafabadi AH, Tamayol A, Annabi N, Ochoa M, Mostafalu P, Akbari M, Nikkhah M, Rahimi R, Dokmeci MR, Sonkusale S, et al.: Biodegradable Nanofibrous Polymeric Substrates for Generating Elastic and Flexible Electronics. Advanced Materials 2014, 33:5823-5830. 
Microfluidics enables fabrication of drug carriers with controlled geometry and release profile.

Microfluidic platforms enable effective localized drug delivery at cell, tissue, and organ level.

Transdermal systems allow easy and painless delivery of active molecules to patients.

Closed-loop smart microfluidic platforms propose the next generation of drug delivery systems. 
(a)

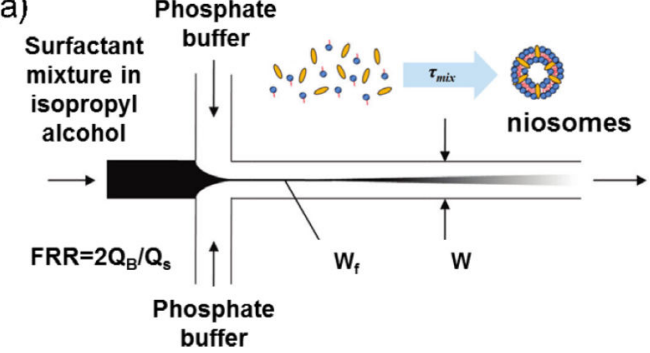

(d)

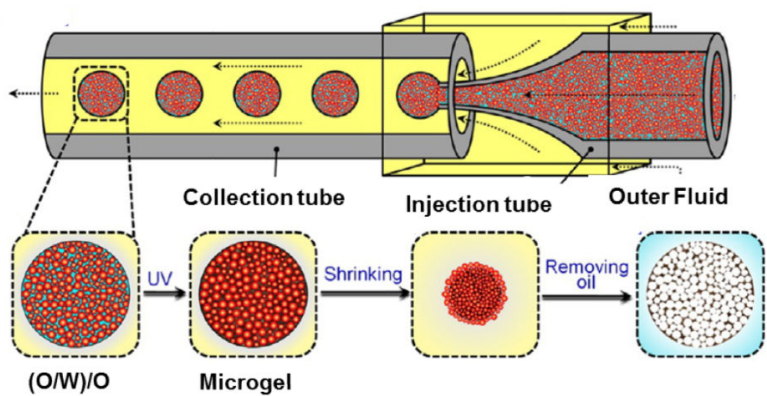

(f)

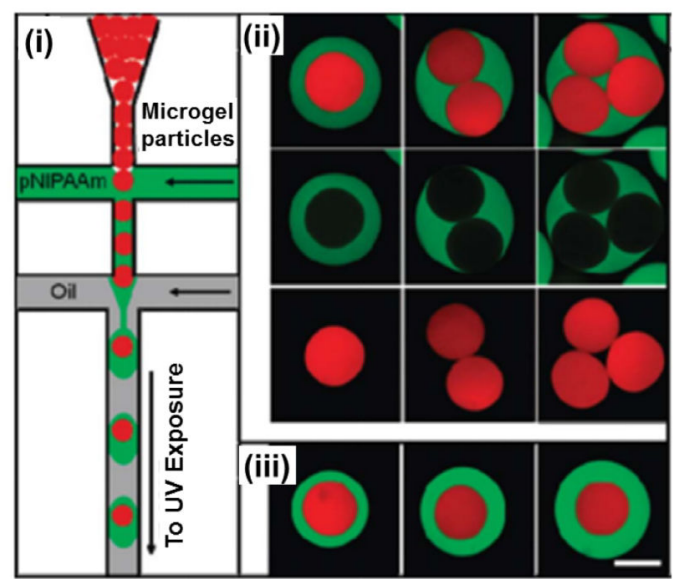

(b)

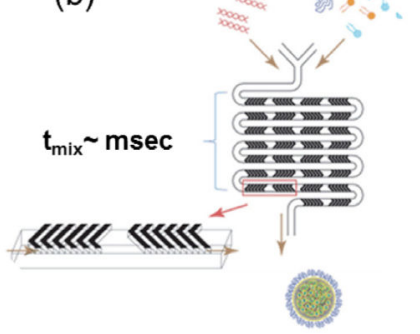

(e)

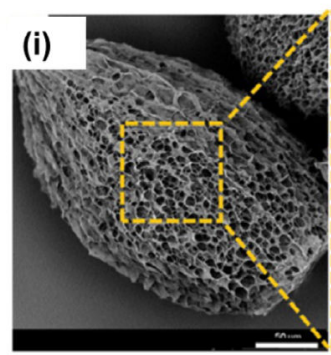

(ii)

(g)

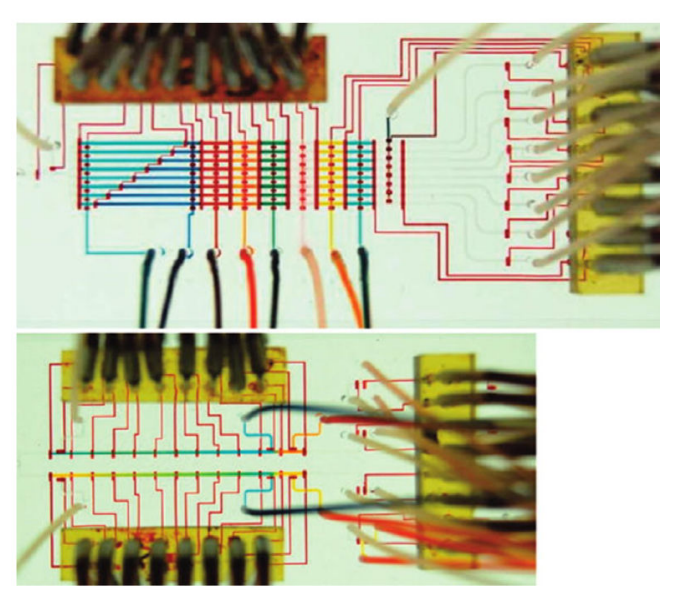

Figure 1.

Microfluidic platforms for production of drug and gene carriers. a) Schematics of niosome self-assembly via HFF in a diffusion-based microfluidic mixer $\left(10 \mathrm{~nm}<D_{p}<100 \mathrm{~nm}\right)$; reprint with permission from[20]. b) Fabrication of lipid nanoparticle (LNP) small interfering RNA (siRNA) formulation strategy employing the staggered herringbone micromixer $(20 \mathrm{~nm}<D p<100 \mathrm{~nm})$; reprint with permission from[29]. c) A multi-inlet microfluidic HFF system to generate lipopolyplex containing Bcl-2 antisense deoxyoligonucleotide (100 nm $<D_{p}<300 \mathrm{~nm}$ ); reprint with permission from[30]. d) Droplet-based microfluidic platform for open-celled porous poly(N-isopropylacrylamide) (PNIPAM) microgel production $(150 \mu \mathrm{m}<D p<450 \mu \mathrm{m})$, e) SEM micrographs of fabricated PNIPAM microgels with open-celled porous structure; reprint with permission from [32] f) Fabrication of microgel capsules that consist of two miscible yet distinct layers using double emulsion template in the droplet-based microfluidic device $\left(20 \mu \mathrm{m}<D_{p}<100\right.$ $\mu \mathrm{m})$; reprint with permission from [6]. g) Programmable microfluidic array for producing a 
combinatorial library of DNA encapsulated supramolecular particles; reprint with permission from $\left(40 \mathrm{~nm}<D_{p}<200 \mu \mathrm{m}\right)$ [44]. 
(a)

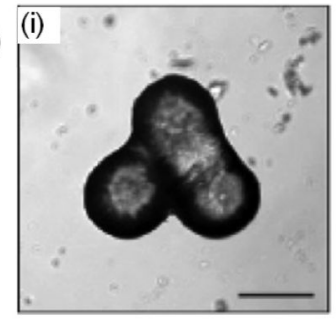

(c)

(i)

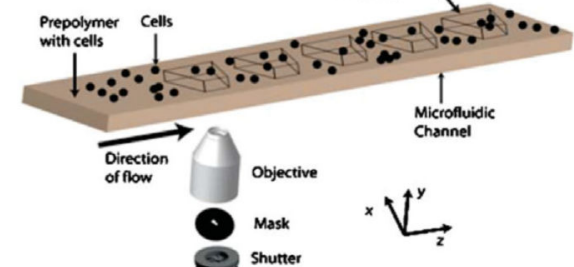

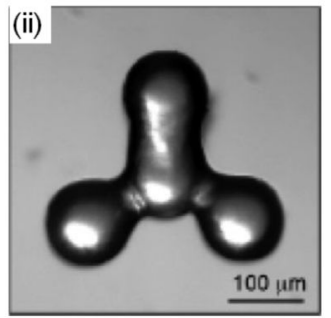

(b)

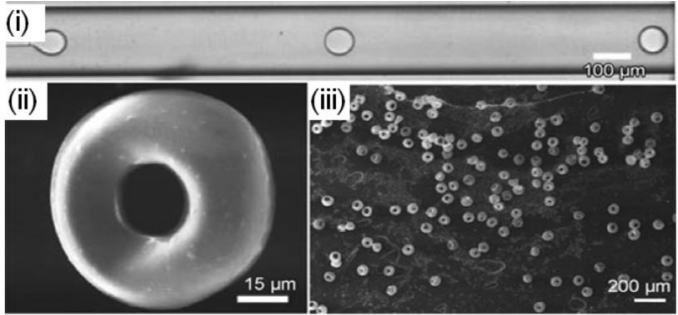

(d)

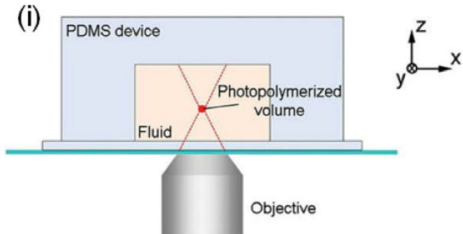

(ii)
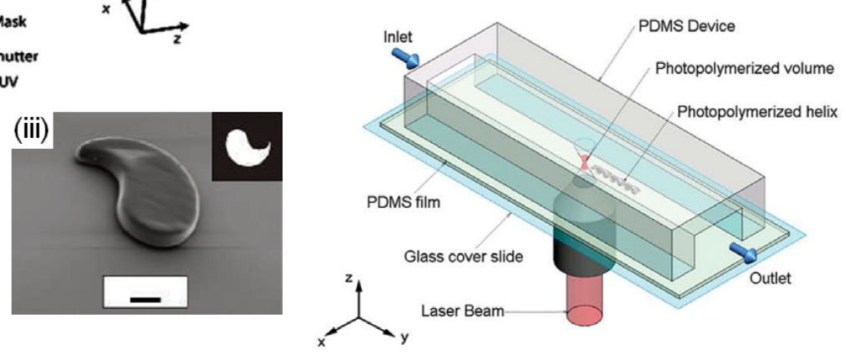

$\overbrace{}^{2}$
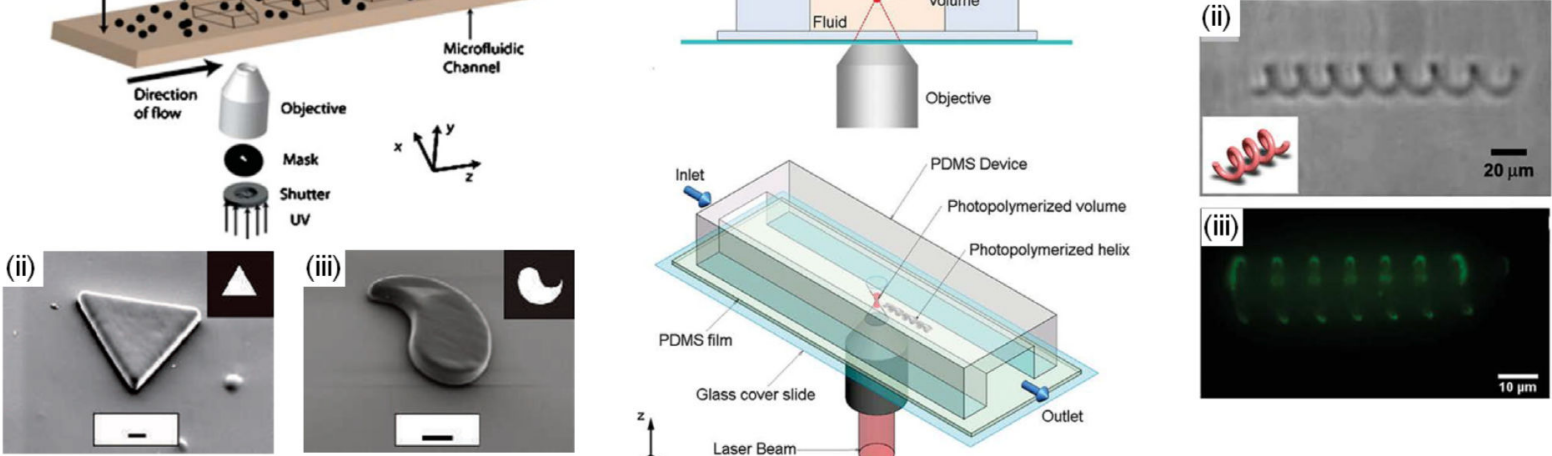

(iii)

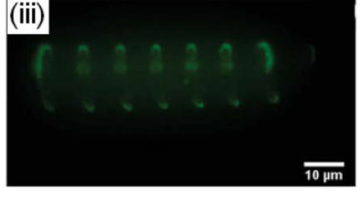

Figure 2.

Microfluidic systems for fabrication of non-spherical particles and carriers. a) nonspherical particles formed by coalescence of spherical particles; reprint with permission from [48]. b) tropoid-like particles fabricated using microfluidic emulsion followed by controlled solvent evaporation; reprint with permission from [49]. c) Stop flow lithography for fabrication of planar particles. (i) schematic of the system, (ii, iii) typical fabricated planar particles using flow lithography; reprint with permission from [52,53]. d) Two-photon continuous flow lithography for fabrication of 3D particles. (i) schematic of the process, (ii) and (iii) bright field and florescent images of a fabricated helical structure; reprint with permission from [54]. 
(a)

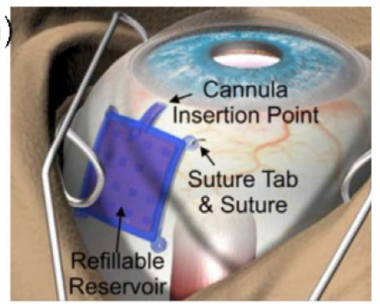

(d)

(g)
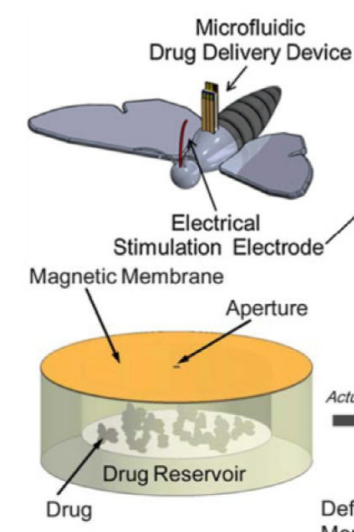

(j)

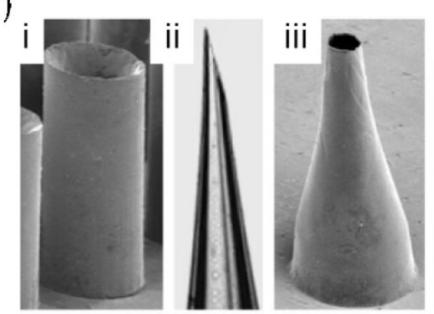

(b)

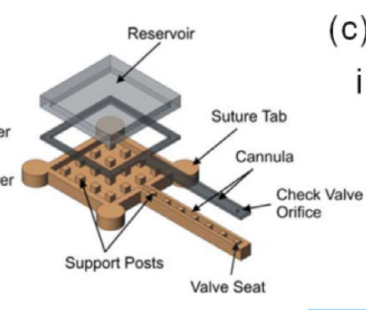

c)

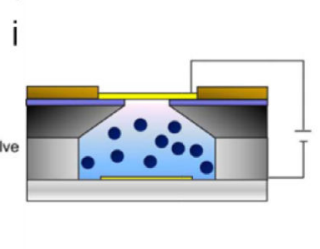

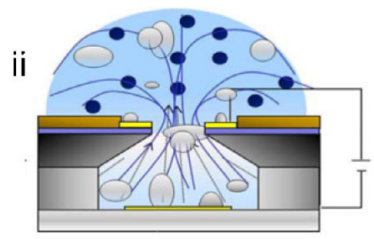

(e)

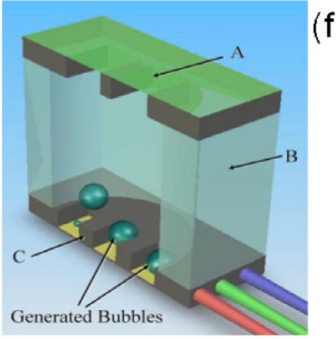

(f)

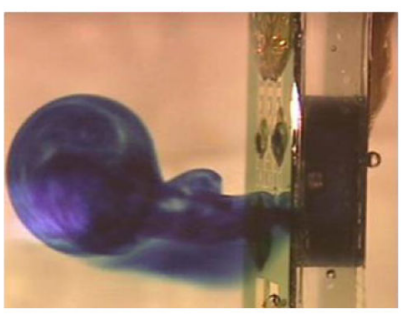

(h)

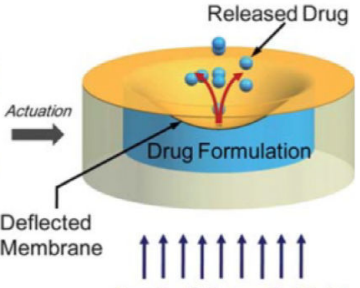

Applied Magnetic Field
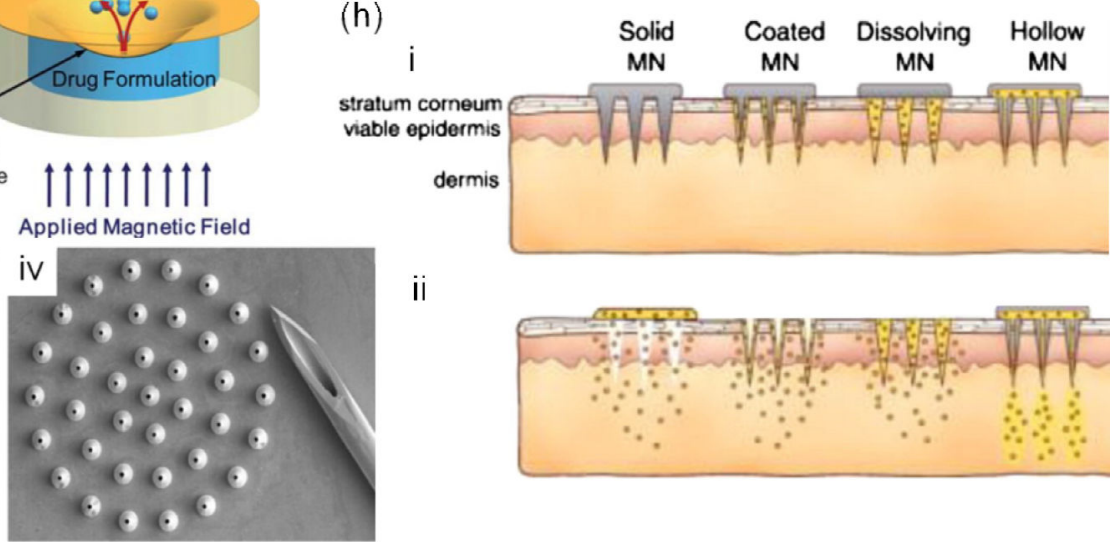

ii

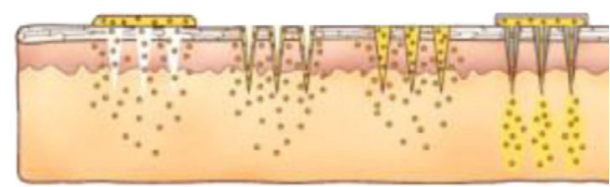

Figure 3.

Microfluidic systems for direct drug delivery. a) Implantation of a microfluidic drug delivery platform for ocular applications, where the drug reservoir was sutured to the sclera and placed underneath the conjunctiva. b) Components of the employed drug delivery platform; reprint with permission from [57]. c) Drug delivery platform that the application of electrical field between the top and bottom electrodes introduced bubbles in the chamber which leads to drug release; reprint with permission from [58]. d) A schematic and an image of a wireless microfluidic system for controlling the flight of Manduca sexta; reprint with permission from [59]. e) A microfluidic drug delivery in which heaters generated bubbles to break the membrane and release the drug, f) Side view of the device illustrating methylene blue release; reprint with permission from[60]. g) Principle of operation of a magnetically actuated drug delivery system; reprint with permission from [61]. h) Various types of microneedle arrays; reprint with permission from [66]. j) SEM images of hollow metal (i,iii) and glass (ii) microneedles. (iv) an array of $500 \mu \mathrm{m}$ long tapered metal microneedles next to a 26-gauge needle; reprint with permission from [62]. 
(a)

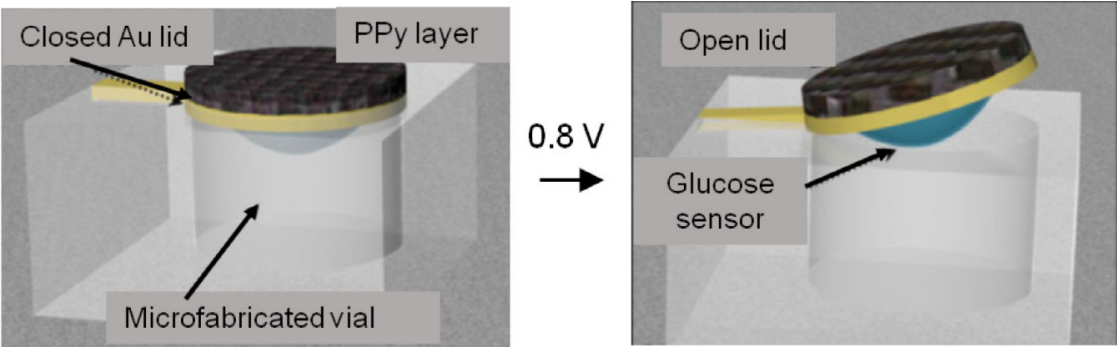

(b)
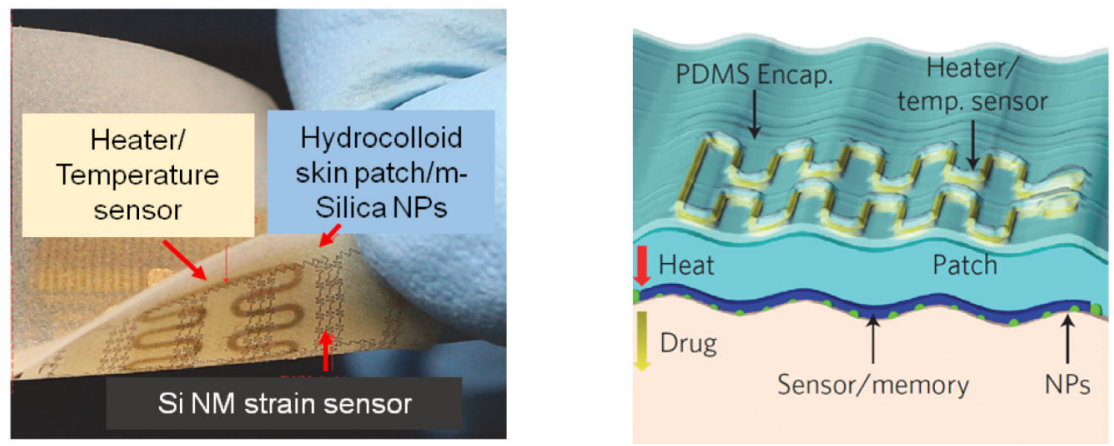

Figure 4.

Smart microfluidic systems for drug delivery. a) schematic diagram of a miniature biosensor immobilized on the backside of the gold lid of a microfabricated vial, which is either closed or open after electroactuation by the application of $800 \mathrm{mV}$ versus $\mathrm{Ag} / \mathrm{AgCl}$; reprint with permission from [68]. b) Controlled transdermal drug delivery from hydrocolloid and $\mathrm{m}$ silica nanoparticles (NPs) by thermal actuation. Wearable electronic patch composed of the data storage modules, diagnostic tools and therapeutic actuating elements; reprint with permission from [70]. 